\title{
Colour Image Segmentation Using Homogeneity Method and Data Fusion Techniques
}

\author{
Salim Ben Chaabane, ${ }^{1}$ Mounir Sayadi, ${ }^{1,2}$ Farhat Fnaiech, ${ }^{1,2}$ and Eric Brassart ${ }^{2}$ \\ ${ }^{1}$ SICISI Unit, High school of sciences and techniques of Tunis (ESSTT), 5 Av. Taha Hussein, 1008 Tunis, Tunisia \\ ${ }^{2}$ Laboratory for Innovation Technologies (LTI-UPRES EA3899), Electrical Power Engineering Group (EESA), \\ University of Picardie Jules Verne, 7, rue du Moulin Neuf, 80000 Amiens, France
}

Correspondence should be addressed to Salim Ben Chaabane, ben_chaabane_salim@yahoo.fr

Received 17 December 2008; Revised 25 March 2009; Accepted 11 May 2009

Recommended by João Manuel R. S. Tavares

\begin{abstract}
A novel method of colour image segmentation based on fuzzy homogeneity and data fusion techniques is presented. The general idea of mass function estimation in the Dempster-Shafer evidence theory of the histogram is extended to the homogeneity domain. The fuzzy homogeneity vector is used to determine the fuzzy region in each primitive colour, whereas, the evidence theory is employed to merge different data sources in order to increase the quality of the information and to obtain an optimal segmented image. Segmentation results from the proposed method are validated and the classification accuracy for the test data available is evaluated, and then a comparative study versus existing techniques is presented. The experimental results demonstrate the superiority of introducing the fuzzy homogeneity method in evidence theory for image segmentation.
\end{abstract}

Copyright (C) 2010 Salim Ben Chaabane et al. This is an open access article distributed under the Creative Commons Attribution License, which permits unrestricted use, distribution, and reproduction in any medium, provided the original work is properly cited.

\section{Introduction}

Image segmentation is considered as an important basic operation for meaningful analysis and interpretation of acquired images $[1,2]$. In this framework, colour image segmentation has wide applications in many areas $[3,4]$, and many different techniques have been developed.

Most published results of colour image segmentation are based on gray level image segmentation methods with different colour representations. Most gray level image segmentation techniques such as histogram thresholding, clustering, region growing, edge detection, fuzzy methods, and neural networks can be extended to colour images. Gray level segmentation methods can be applied directly to each component of a colour space, and then the results can be combined in some way to obtain a final segmentation result.

In the Red, Green, Blue (RGB) representation, the colour of each pixel is usually represented on the basis of the three primary colours (red, green, and blue), but it can be coded in other representation systems which are grouped together according to their different properties. RGB is suitable for colour display, but inappropriate for colour scene segmentation and analysis because of the high correlation among the R, G, and B components [5]. In this context, image segmentation using data fusion techniques appears to be an interesting method.

Data fusion is a technique which simultaneously takes into account heterogeneous data coming from different sources, in order to obtain an optimal set of objects for investigation. Of the existing data fusion methods such as probability theory [6], fuzzy logic [7-9], possibility theory [10], evidence theory $[11,12$, the Dempster-Shafer (DS) evidence theory [13], is a powerful and flexible mathematical tool for handling uncertain, imprecise, and incomplete information. In the case of evidence theory, the determination of mass function is a crucial step of fusion process.

In the past, many authors have addressed this problem using different methods [14-17], and several researchers have, in particular, investigated the relationship between fuzzy sets and Dempster-Shafer evidence theory. Most of the literature using fuzzy sets has been focused on automatically determining the mass function in the DS evidence theory $[17,18]$. Recently, most analytic fuzzy methods have been 
derived from Bezdek's Fuzzy C-Means (FCM) [19, 20]. However, this algorithm has a considerable drawback in noisy environments, and the degrees of membership resulting from FCM do not correspond to the intuitive concept of belonging or compatibility. Also, the Hard C-Means (HCM) [21] is one of the oldest clustering methods in which HCM memberships are hard (i.e., 1 or 0 ). This method is used to learn the prototypes of clusters or classes, and the cluster centers are used as prototypes.

In this context, Gautier et al. [22] aim at providing a help to the doctor for the follow-up of the diseases of the spinal column. The objective is to rebuild each vertebral lumbar rachis starting from a series of cross-sections. From an initial segmentation obtained by using the Snakes, active contour models, one seeks a segmentation which represents as well as possible the anatomical contour of the vertebra, in order to give the doctors a schema of the points really forming part of the vertebra. The methodology is based on the application of the belief theory to fusion information. However, the active contour models do not require image preprocessing and provide a closed contour of the object, however typical problems remain difficult to solve including the initialization of the model.

With the same objective, Zimmermann and Zysno [14], have shown through empirical studies that the good Model for Membership Functions is based on the Distance of a point from a prototypical member (MMFD). However, one of the major factors that influences the determination of appropriate groups of points is the "distance measure" chosen for the problem at hand. Also, Zhu et al. [17], and Ben Chaabane et al. [23] have proposed a method of automatically determining the mass function for image segmentation problems. The idea is to assign, at each image pixel level, a mass function that corresponds to a membership function in fuzzy logic. The degrees of membership of each pixel are determined by applying fuzzy c-means (FCM) clustering to the gray levels of the image.

In another study, Vannoorenberghe et al. [16] and Ben Chaabane et al. [24] have proposed an information model obtained from training sets extracted from the pixel intensity of the image. In their papers, the authors described the estimation of the Model Mass Function method based on the Assumption of Gaussian Distribution (MMFAGD) and histogram thresholding and applied on synthetic and biomedical images that contain only two classes. However, the differences between the various works cited above occur in the method of mass functions estimation, and in the application.

In this paper an investigation of how the user can choose the best a priori knowledge for determining the mass function in Dempster-Shafer evidence theory is described. We shall assume a Gaussian distribution for estimating the mass function. So, this work may be seen to be straightforwardly complementary to that in the paper proposed by Vannoorenberghe et al. [16] and Ben Chaabane et al. [24]. In their paper, the authors suggested that the user has to search for a suitable method for determining the a priori knowledge. Hence, this paper is devoted to this task, applied to colour image segmentation that contains more than two classes. The idea is based on the histogram thresholding of the homogeneity and data fusion techniques. The concept of the histogram of the homogeneity was discussed in [25], which is used to express the local and global information among pixels in an image. Histogram analysis is applied to find all major homogeneous regions in the three primitive colours. The assumption of a Gaussian distribution is used to calculate the mass function of each pixel. Once the mass functions are determined for each primitive colour to be fused, the DS combination rule and decision are applied to obtain the final segmentation.

Section 2 introduces the proposed method for colour image segmentation. The experimental results are discussed in Section 3, and the conclusion is given in Section 4 .

\section{Proposed Method}

For colour images with RGB representation, the colour of a pixel is a mixture of the three primitive colours red, green, and blue. RGB is suitable for colour display, but not good for colour scene segmentation and analysis because of the high correlation among the R, G, and B components $[5,26]$. By high correlation, we mean that if the intensity changes, all the three components will change accordingly. In this context, colour image segmentation using evidence theory appears to be an interesting method. However, to fuse different images using DS theory, the appropriate determination of mass function plays a crucial role, since assignation of a pixel to a cluster is given directly by the estimated mass functions. In the present study, the method of generating the mass functions is based on the assumption of a Gaussian distribution. To do this, histogram analysis is applied simultaneously to both the homogeneity and the colour feature domains. These are used to extract homogeneous regions in each primitive colour. Once the mass functions are estimated, the DS combination rule is applied to obtain the final segmentation results.

2.1. Homogeneity Histogram Analysis. Histogram thresholding is one of the widely used techniques for monochrome image segmentation, but it is based on only gray levels and does not take into account the spatial information of pixels with respect to each other. A comprehensive survey of image thresholding methods is provided in [27]. Cheng et al. [25, $28,29]$, proposed a fuzzy homogeneity method to overcome this limitation. In this paper, we employ the concept of the homogeneity histogram to extract homogeneous regions in each primitive colour.

Assume $g_{x y}$ is the intensity of a pixel $p_{x y}$ at the location $(x, y)$ in an $(M \times N)$ image, $w_{x y}^{(1)}$ is a size $(d \times d)$ window centered at $(x, y)$ for the computation of variation, $w_{x y}^{(2)}$ is a size $(t \times t)$ window centered at $(x, y)$ for the computation of discontinuity. Let us choose a $5 \times 5$ window for computing the standard deviation of a pixel $p_{x y}$, and a $3 \times 3$ window for computing the edge. However, $w_{x y}^{(1)}$ and $w_{x y}^{(2)}$ are the 
local regions where the homogeneity features for pixel are calculated. By assuming, that the signals are ergodic, the standard deviation describes the contrast within a local region [30], and is calculated for a pixel $p_{x y}$ as follows:

$$
v_{x y}=\sqrt{\frac{1}{d^{2}} \sum_{p=x-(d-1) / 2}^{x+(d-1) / 2} \sum_{q=y-(d-1) / 2}^{y+(d-1) / 2}\left(g_{p q}-\mu_{x y}\right)^{2}},
$$

where $x \geq 2, p \leq M-1, y \geq 2$, and $q \leq N-1$.

$\mu_{x y}$ is the mean of the gray levels within window $w_{x y}$ and is defined by:

$$
\mu_{x y}=\frac{1}{d^{2}} \sum_{p=x-(d+1) / 2}^{x+(d-1) / 2} \sum_{q=y-(d+1) / 2}^{y+(d-1) / 2} g_{p q} .
$$

The discontinuity is a measure of abrupt changes in gray levels of pixels $p_{x y}$, that is, the discontinuity is described by its edge value, and could be obtained by applying edge detectors to the corresponding region. There are many different edge operators: Sobel, Canny, Derish, Laplace, and so forth, but their functions and performances are not the same. In spite of all the efforts, none of the proposed operators are fully satisfactory in real world cases. Applying different operators to a noisy image shows that, the second derivative operators exhibit better performance than classical operators, but require more computations because the image is first smoothed with a Gaussian function and then the gradient is computed [31]. Liu and Haralick [32] have evaluated the performance of edge detection algorithms.

Since it is not necessary to find the accurate locations of the edges, and due to its simplicity, the Sobel operator for calculating the discontinuity and the magnitude of the gradient at location $(x, y)$ are used for their measurement [30]:

$$
c_{x y}=\sqrt{G_{x^{\prime}}^{2}+G_{y^{\prime}}^{2}}
$$

where $G_{x^{\prime}}$ and $G_{y^{\prime}}$ are the components of the gradient in the $x^{\prime}$ and $y^{\prime}$ directions, respectively.

The homogeneity is represented by:

$$
h\left(g_{x y}, w_{x y}^{(1)}, w_{x y}^{(2)}\right)=1-E\left(g_{x y}, w_{x y}^{(2)}\right) \times V\left(g_{x y}, w_{x y}^{(1)}\right),
$$

where

$$
\begin{aligned}
& V\left(g_{x y}, w_{x y}^{(1)}\right)=\frac{v_{x y}}{\max \left\{v_{x y}\right\}}, \\
& E\left(g_{x y}, w_{x y}^{(2)}\right)=\frac{c_{x y}}{\max \left\{c_{x y}\right\}},
\end{aligned}
$$

$c_{x y}$ and $v_{x y}$ are, respectively, the discontinuity and the standard deviation of a pixel $p_{x y}$ at the location $(x, y), 2 \leq$ $x \leq M-1$ and $2 \leq y \leq N-1$.

However, the size of the windows has an influence on the calculation of the value of the homogeneity. The window should be big enough to allow enough local information about the pixel to be involved in the computation of the homogeneity. Furthermore, using a larger window in the computation of the homogeneity increases the smoothing effect, and makes the derivative operations less sensitive to noise [13]. However, smoothing the local area might hide some abrupt changes of the local region. Also, a large window causes significant processing time. In our case, the sizes of the windows are selected experimentally over 120 images. Weighting the pros and cons, experimentally, a $5 \times 5$ window for computing the standard deviation of the pixel, and a $3 \times 3$ window for computing the edge are chosen.

Once the homogeneity histogram has been determined, a typical segmentation method based on histogram analysis is applied to each primitive colour. Sezgin and Sankur [27] have examined and evaluated the quantitative performance of several thresholding techniques. Finally, a peak finding algorithm whose general form is reviewed as follows [25].

Input an $M \times N$ image with gray levels zero to 255 .

Suppose a homogeneity histogram of an image represented by a function $h(i)$, where $i$ is an integer, $0 \leq i \leq 255$ and the value of the homogeneity at each location of an image has a range from $[0,1]$.

Step 1. Find the set of points $p_{i}$ corresponding to the local maximums of the histogram.

The result forms a set $P_{0}$ :

$$
\begin{gathered}
P_{0}=\{(i), h(i) \mid h(i)>h(i-1), h(i)>h(i+1), \\
1 \leq i \leq 254\} .
\end{gathered}
$$

Step 2. Find significant peaks in set $P_{0}$.

The result form the set $P_{1}$ :

$$
\begin{aligned}
P_{1}= & \left\{\left(p_{i}\right), h\left(p_{i}\right) \mid h\left(p_{i}\right)>h\left(p_{i-1}\right), h\left(p_{i}\right)>h\left(p_{i+1}\right),\right. \\
& \left.p_{i} \in P_{0}\right\} .
\end{aligned}
$$

Step 3. Thresolding: includes three substeps.

(i) Remove small peaks: for any peak $j$, if $(h(i) /$ $\left.h\left(i_{\max }\right)\right)<0.05$, then the peak $j$ is removed, where $i_{\max }$ is the value of the highest peak.

(ii) Choose one peak among two peaks $\left(p_{1}\right.$ and $p_{2}$ ) if they are too close to each others.

If $\left(p_{2}-p_{1}\right) \leq 12$ then $h=\max \left(h\left(p_{1}\right), h\left(p_{2}\right)\right)$.

(iii) Remove a peak if the valley between two peaks is not significant.

Comments. The first substep below Step 2.1 related to the thresholding is used for removing the small peaks compared with the biggest. For any peak $j$, if $\left(h(i) / h\left(i_{\max }\right)\right)<0.05$, then peak $j$ is removed. The threshold 0.05 is based on the experiments over more than 120 images. Since the value of the homogeneity at each location of an image has a range from $[0,1], h\left(i_{\max }\right)$ is equal to 1 . Therefore, the points with $h\left(i_{\max }\right)<0.05$ will be removed.

The second substep below Step 2.1 is to select one peak from two peaks close to each other. For two peaks $h\left(p_{1}\right)$ and $h\left(p_{2}\right), p_{2}>p_{1}$, if $\left(p_{2}-p_{1}\right) \leq 12$, then $h=\max \left(h\left(p_{1}\right), h\left(p_{2}\right)\right)$. Thus, the peak with the biggest value is chosen. 
Finally, the third substep of Step 2.1 is applied for removing a peak if the valley between two peaks is not significant. The valley is not deep enough to separate the two peaks, if $h_{\mathrm{aver}_{1}} / h_{\mathrm{aver}_{2}}>0.75$, where $h_{\mathrm{aver}_{1}}$ is the average value among the points between peaks $p_{1}$ and $p_{2}$ indicated by

$$
h_{\mathrm{aver}_{1}}=\frac{\sum_{p_{i}=p_{1}}^{p_{i}=p_{2}} h\left(p_{i}\right)}{p_{2}-p_{1}+1}
$$

and $h_{\text {aver }}$ is the average value for the two peaks defined by

$$
h_{\mathrm{aver}_{2}}=\frac{h\left(p_{1}\right)+h\left(p_{2}\right)}{2} .
$$

The distance 12 between two peaks is selected experimentally over 120 images. It is a minimum threshold used to choose one of these two peaks. Also, the threshold 0.75 is based on the experiments over than 120 images.

\subsection{Use of DS Evidence Theory for Image Segmentation.}

The purpose of segmentation is to partition the image into homogeneous regions. The idea of using DS evidence theory for image segmentation is to fuse one by one the pixels coming from the three images. The homogeneity method is applied to the three primitive colours. Then, the segmented results are combined using the Dempster-Shafer evidence theory to obtain the final segmentation results.

Dempster-Shafer Theory (DS) is a mathematical theory of evidence [11, 12]. This theory can be interpreted as a generalization of probability theory where probabilities are assigned to sets as opposed to mutually exclusive singletons. In traditional probability theory, evidence is associated with only one possible event.

In DS theory, evidence can be associated with multiple possible events, for example, sets of events. One of the most important features of Dempster-Shafer theory is that the model is designed to cope with varying levels of precision regarding the information.

In the present study, the clusters $\left(C_{i}\right)$ are generated by the homogeneity method from the frame of discernment $\Omega$ composed of $n$ single mutually exclusive subsets $H_{n}$, which are symbolized by

$$
\Omega=\left\{H_{1}, H_{2}, \ldots, H_{n}\right\}=\left\{C_{i}\right\}, \quad 1 \leq i \leq n .
$$

In order to express a degree of confidence for each proposition $A$ of $2^{\Omega}$, it is possible to associate an elementary mass function $m(A)$ which indicates the degree of confidence that one can give to this proposition. Formally, this description of $m$ can be represented with the following three equations:

$$
\begin{gathered}
m: 2^{\Omega} \longrightarrow[0,1], \\
m(\phi)=0, \\
\sum_{A_{n} \subseteq \Omega} m(A)=1 .
\end{gathered}
$$

The quantity $m(A)$ is interpreted as the belief strictly placed on $A$. This quantity differs from a probability by the totality of the total belief which is distributed not only on the simple classes but also on the composed classes. This modelling shows the impossibility of dissociating several hypotheses. Hence, it is the principal advantage of this theory, but on the other hand, it represents the main difficulty of this method.

In the following, we give some useful definitions. In fact if $m(A)>0$ then $A$ is called a focal element.

The union of all the focal elements of a mass function is called the core $N$ of the mass function given by the following equation:

$$
N=\left\{A \in 2^{\Omega} / m(A)>0\right\} .
$$

Credibility $\mathrm{Cr}(\cdot)$ and plausibility $\mathrm{Pl}(\cdot)$ functions are derived from the mass function. However, the credibility for a set $H_{n}$ is defined as the sum of all the basic probability assignments of the proper subsets $(A)$ of the set of interest $\left(H_{n}\right)\left(A \subseteq H_{n}\right)$, see (13). The value $\operatorname{Cr}\left(H_{n}\right)$ denotes the minimal degree of belief in the hypothesis $H_{n}$ :

$$
\operatorname{Cr}\left(H_{n}\right)=\sum_{A \subseteq H_{n}} m(A) .
$$

The Plausibility is the sum of all the basic probability assignments of the sets $(A)$ that intersect the set of interest $\left(H_{n}\right)\left(A \cap H_{n}=\phi\right)$, see (14). The value $\operatorname{Pl}\left(H_{n}\right)$ gives the maximal degree of belief in the hypothesis $H_{n}$ :

$$
\operatorname{Pl}\left(H_{n}\right)=\sum_{A \cap H_{n} \neq \phi} m(A) .
$$

The Dempster rule of combination is critical to the original concept of Dempster-Shafer theory. Dempster's rule combines multiple belief functions through their basic probability assignments $(m)$. These belief functions are defined on the same frame of discernment, but are based on independent arguments or bodies of evidence. The combination rule results in a belief function based on conjunctive-pooled evidence.

The combination is performed by the orthogonal sum of Dempster, and is expressed for $n$ sources as

$$
\begin{aligned}
\bigoplus_{i=1}^{n} & m_{i}\left(H_{n}\right) \\
& =\frac{1}{1-k} \sum_{A_{1} \cap A_{2} \cap \cdots \cap A_{n}=H_{n}} m_{1}\left(A_{1}\right) m_{2}\left(A_{2}\right) \cdots m_{n}\left(A_{n}\right),
\end{aligned}
$$

where $H_{n}, A_{1}, \ldots, A_{n}$ are subsets of $\Omega$, and

$$
k=\sum_{A_{1} \cap A_{2} \cap \cdots \cap A_{n}=\phi} m_{1}\left(A_{1}\right) m_{2}\left(A_{2}\right) \cdots m_{n}\left(A_{n}\right) .
$$

Specifically, the combination (called the joint $m_{12}$ ) is calculated from the aggregation of two mass functions $m_{1}$ and $m_{2}$ and given as follows:

$$
\forall H_{i} \subseteq \Omega, \quad m_{12}\left(H_{i}\right)=\frac{1}{1-K} \sum_{A_{1} \cap A_{2}=H_{i}} m_{1}\left(A_{1}\right) m_{2}\left(A_{2}\right),
$$


where $K$ is defined by [11]:

$$
K=\sum_{A_{1} \cap A_{2}=\phi} m_{1}\left(A_{1}\right) m_{2}\left(A_{2}\right),
$$

$K$ represents the basic probability mass associated with conflict. This is determined by summing the products of mass functions of all sets where the intersection is an empty set.

This rule is commutative and associative. The denominator in Dempster's rule, $(1-K)$, is a normalization factor, which evaluates the conflict between the two sources $A_{1}$ and $A_{2}$.

The DS theory of evidence is a rich model of uncertainty handling as it allows the expression of partial belief [9].

2.2.1. Mass Function of Simple Hypotheses. Masses of simple hypotheses $C_{i}$ are obtained from the assumption of Gaussian Distributions of the grey level $g_{x y}$ to cluster $i$ as follows:

$$
m_{q}^{x y}\left(C_{i}\right)=\frac{1}{\sigma_{i} \sqrt{2 \pi}} \exp \frac{-\left(g_{x y}^{q}-\mu_{i}\right)^{2}}{2 \sigma_{i}^{2}},
$$

where $g_{x y}^{q}$ is the intensity of a pixel $p_{x y}$ at the location $(x, y)$ for one of the three information sources $(q=1,2,3)$. The values $\mu_{i}=E\left(g_{x y}^{q}\right)$ and $\sigma_{i}^{2}=E\left(g_{x y}^{q}-E\left(g_{x y}^{q}\right)\right)^{2}$ are, respectively, the mean and the variance on the class $C_{i}$ present in each primitive colour (R, G, and B). E denoted the mathematical expectation.

2.2.2. Mass Function of Double Hypotheses. The mass function assigned to double hypotheses depends on the mass functions of each hypothesis.

In fact, if there is a high ambiguity in assigning a grey level $g_{x y}$ to cluster $r$ or $s$, that is, $\left|m_{q}^{x y}\left(C_{r}\right)-m_{q}^{x y}\left(C_{s}\right)\right|<\varepsilon$, where $\varepsilon$ is the thresholding value, then a double hypotheses is formed. In the present study, $\varepsilon$ was fixed at 0.1 .

Once the double hypotheses (composed of two simple hypotheses) are formed, their joint mass is calculated according to the following formula:

$$
m_{q}^{x y}\left(C_{r} \cup C_{s}\right)=\frac{1}{\sigma_{r s} \sqrt{2 \pi}} \exp \frac{-\left(g_{x y}^{q}-\mu_{r s}\right)^{2}}{2 \sigma_{r s}^{2}},
$$

with $\mu_{r s}=\left(\mu_{r}+\mu_{s}\right) / 2$ and $\sigma_{r s}=\max \left(\sigma_{r}, \sigma_{s}\right)$.

In the case where the double hypotheses $C_{j}$ are composed of more than two simple hypotheses, their joint mass is determined as follows:

$$
m_{q}^{x y}\left(C_{1} \cup C_{2} \cup \cdots \cup C_{M}\right)=\frac{1}{\sigma_{j} \sqrt{2 \pi}} \exp \frac{-\left(g_{x y}^{q}-\mu_{j}\right)^{2}}{2 \sigma_{j}^{2}},
$$

where $\mu_{j}=(1 / M) \sum_{i=1}^{M} \mu_{i}, \sigma_{j}=\max \left(\sigma_{1}, \sigma_{2}, \ldots, \sigma_{M}\right)$ and $2<$ $M \leq N$.

Once the mass functions of the three images are estimated, their combination is performed using the orthogonal sum that can be represented as follows:

$$
m\left(C_{i}\right)=m_{1}\left(C_{i}\right) \oplus m_{2}\left(C_{i}\right) \oplus m_{3}\left(C_{i}\right)
$$

with $\oplus$ is the sum of DS orthogonal rule.
After calculating the orthogonal sum of the mass functions for the three images, the decisional procedure for classification purpose consists in choosing one of the most likely hypotheses $C_{i}$. The proposed method can be described by a flowchart given in Figure 1.

\section{Experimental Results}

In this section, several results of the simulations on the segmentation of medical and synthetic colour images (Figure 5), which illustrate the ideas presented in the previous section, are given.

In order to evaluate the performance of the proposed algorithm on the segmentation of colour cell images (which is a challenging problem in this field), the segmentation results of the datasets are reported. Consequently, a synthetic image dataset is developed and used for numerical evaluation purpose.

First the segmentation results in RGB colour space by applying the proposed method to red, green, and blue colour features, respectively, are presented. In this case, we find that the regions are recognized for example in red and green components but are not identified by the blue component. This shows the lack of information when using only one information source and may be explained by the high degree of correlation among of the three components of the RGB colour space.

The experimentation is carried out on a medical image provided by a cancer hospital Figure 2(a) and used as an original image. The results are shown in Figures 2(b), 2(c), and $2(\mathrm{~d})$.

The problem of the incorrectly segmentation is also illustrated in Figure 2(b), the resulting image has four cells, while in Figures 2(c) and 2(d) the resulting image by using homogeneity histogram thresholding has only three and two cells, respectively.

Comparing the results, we can find that the cells are much better segmented in (b) than in (c) and (d). Also, the first resulting image contains some missing features in one of the cells, which do not exist in the other resulting images. This demonstrates the necessity of using the fusion process

Also let us compare the performance of our proposed algorithm to those in other published reports that have recently been applied to colour images. These include Zimmermann and Zysno [14], Vannoorenberghe et al. [16], Ben Chaabane et al. [24], Zhu et al. [17], and Ben Chaabane et al. [23].

The segmentation results are shown in Figures 3, 4, 6, and 7.

Firstly let us present a colour image that contains two classes. To highlight its performance let us compare it with the MMFD [14] and MMFAGD [24] algorithms.

Secondly let us work on more realistic images containing multiple classes and compare the performance of our method with other methods that use FCM [23], and HCM [21] algorithms as tools for the estimation of mass functions in the Dempster-Shafer evidence theory. Figures 3, 4, 6, and 7 show the obtained results of the proposed method. 


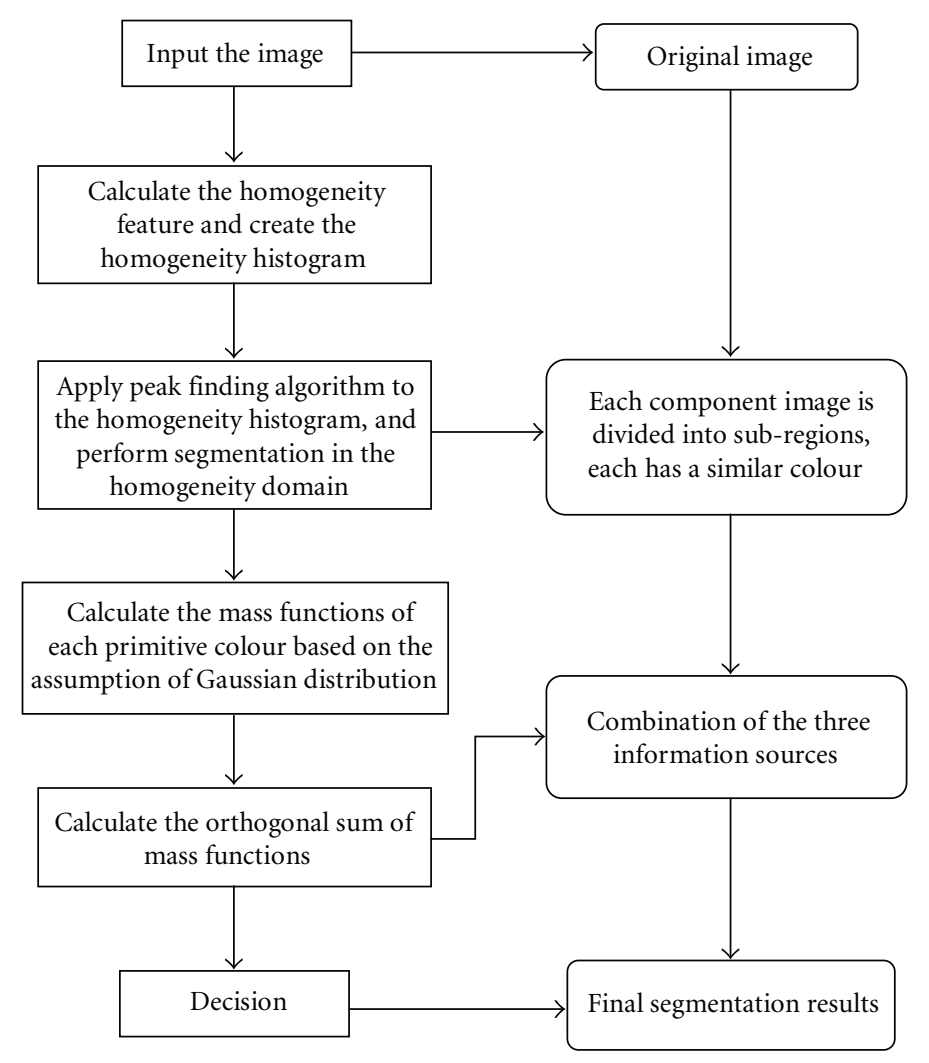

FIgURE 1: Flowchart of the proposed method.

The original images are artificial, that is, generated with a user defined classification, and are stored in RGB format. Each of the primitive colours (red, green, and blue) takes 8 bits and has an intensity range from 0 to 255 .

Figure 3 shows a comparison of the results between the traditional methods MMFD [14], MMFADG [24], and the proposed method. However, the image shown in Figure 3(b) represents the original image $I$ where a "Salt and pepper" noise of $D$ density was added. This affects approximately $(D \times(N \times M))$ pixels. The value of $D$ is 0.02 .

The final images using the MMFD and MMFAGD algorithms and the homogeneity for the determination of mass functions in DS theory are shown in Figures 3(c), 3(d), and 3(e), respectively.

Comparing Figures 3(c), 3(d), and 3(e), one can find that the cell is much better segmented in (e) than those in (c) and (d), also the first and the second images contain some holes in the cell and some pixels were incorrectly segmented. These do not exist in the correctly segmented image, but after the redefining process, only a few singularity points are left in the final image as shown in Figure 3(e).

Accordingly, the dark blue colour of the cell is identified by the proposed method (Figure 3(e)), but is not seen in other traditional methods (Figures 3(c) and 3(d)).

It can be seen from Table 1 that $31.77 \%, 20.44 \%$, and $2.73 \%$ of pixels were incorrectly segmented in Figures 3(c), $3(\mathrm{~d})$, and 3(e), respectively. However, the two regions are correctly segmented in Figure 3(e), using the complementary information provided by the three primitive colours and consequently a good estimation of mass function by homogeneity, even in the presence of a noise (without the filtering step) is recorded.

In fact, the experimental results indicate that the proposed method, which uses both local and global information for mass function calculation in DS evidence theory, is more accurate than the traditional methods in terms of segmentation quality as denoted by segmentation sensitivity, see Table 1 .

In the method based on traditional histogram thresholding $[16,24]$ only global information is considered in the histogram analysis.

To provide insights into the proposed method, we have compared the performance of the proposed method with those of the corresponding Hard and Fuzzy CMeans algorithms. The method was also tested on synthetic images and compared with other existing methods, see Figure 4.

Figure 4 shows a synthetic input image that contains a multicomponent object with complicated boundaries and different component sizes. This figure consists of mainly six kinds of objects. After applying the HCM and FCM algorithms for the estimation of the mass function in DS evidence theory, followed by the data fusion techniques, the resulting image is divided into only four and five regions, respectively. But, using the proposed segmentation method, the resulting image is divided into six regions. 
TABLE 1: Segmentation sensitivity from MMFD and DS, MMFAGD and DS and homogeneity method and DS for the data set shown in Figure 5.

\begin{tabular}{|c|c|c|c|}
\hline & MMFD and DS & MMFAGD and DS & $\begin{array}{c}\text { Homogeneity and DS } \\
\text { (proposed method) }\end{array}$ \\
\hline & \multicolumn{3}{|c|}{ Sensitivity segmentation (\%) } \\
\hline Image 1 & 66.84 & 72.94 & 94.23 \\
\hline Image 2 & 68.23 & 79.66 & 97.27 \\
\hline Image 3 & 72.56 & 83.19 & 90.84 \\
\hline Image 4 & 85.11 & 88.91 & 98.11 \\
\hline Image 5 & 75.42 & 76.86 & 96.85 \\
\hline Image 6 & 63.71 & 81.45 & 98.58 \\
\hline Image 7 & 83.54 & 93.88 & 98.36 \\
\hline Image 8 & 66.78 & 79.33 & 95.37 \\
\hline Image 9 & 75.84 & 77.85 & 99.85 \\
\hline Image 10 & 54.85 & 75.17 & 96.97 \\
\hline Image 11 & 62.74 & 74.43 & 81.13 \\
\hline Image 12 & 45.37 & 68.45 & 97.72 \\
\hline
\end{tabular}

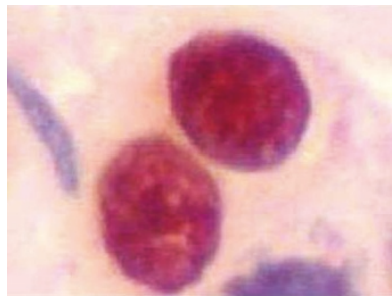

(a)

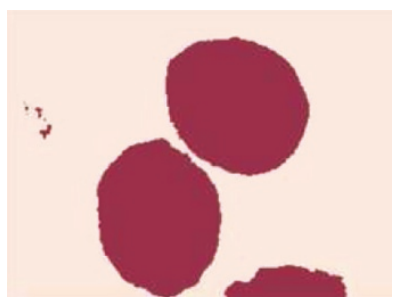

(c)

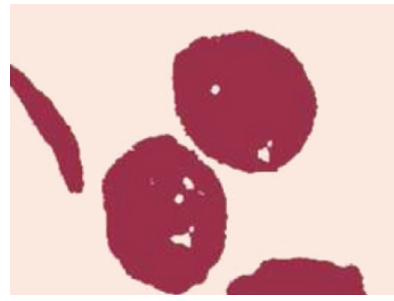

(b)

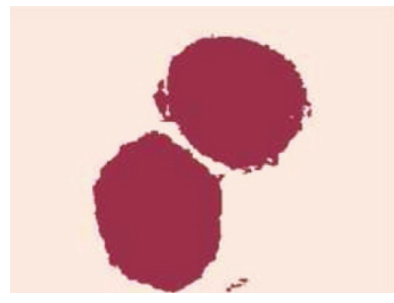

(d)
FIgURe 2: Segmentation results on a colour image. (a) Original image $(256 \times 256 \times 3)$ with gray level spread on the range $[0,255]$. (b) Red resulting image by homogeneity histogram-based method. (c) Green resulting image by homogeneity histogram-based method. (d) Blue resulting image by homogeneity histogram-based method. The selected thresholds are 147, 110, and 194, respectively.

In brief, the experimental results conform to the visualized colour distribution in the objects. However, the new classes that appeared in Figure 6(d), tend to increase the size of some regions (yellow regions), and to shrink other regions (flowers), and some incorrectly segmented pixels are present in Figure 6(c), such as the extra blue contouring in the bottom centre flower.

The improved experimental results have been achieved by the proposed method based on the homogeneity histogram which can be used to generate a mass function that has a typical interpretation, that is, the resulting partition

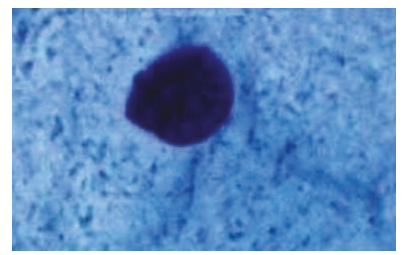

(a)

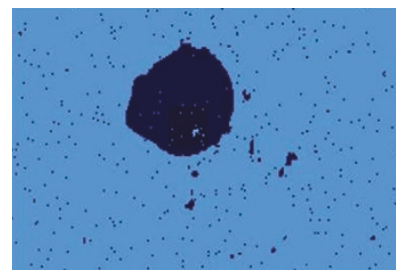

(c)

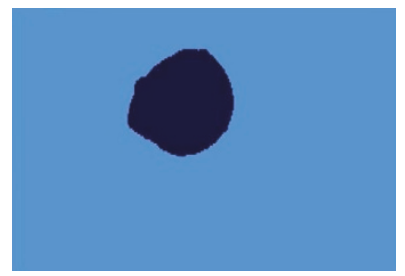

(e)

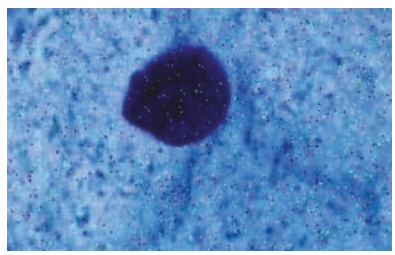

(b)

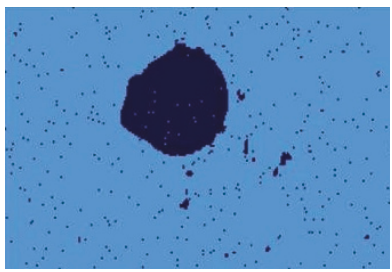

(d)

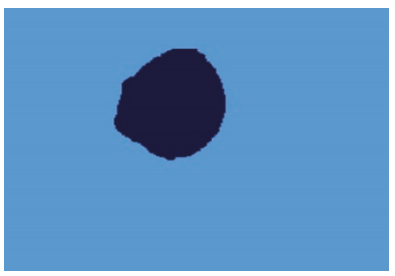

(f)
FIGURE 3: Comparison of the proposed segmentation method with other existing methods on a medical image ( 2 classes, 1 cell). (a) Original image with RGB representation $(256 \times 256 \times 3)$, (b) colour cell image disturbed with a "salt and pepper" noise, (c) segmentation based on MMFD and DS (d) segmentation based on MMFAGD and DS, (e) segmentation based on homogeneity and DS, and (f) reference segmented image.

of the data can be interpreted as the compatibilities of the points with the class prototypes, while the HCM and FCM methods use only the gray level to determine the degree of membership of each pixel. 
TABLE 2: Segmentation sensitivity from HCM and DS, FCM and DS and homogeneity method and DS for the data set shown in Figure 5.

\begin{tabular}{lccc}
\hline & HCM and DS & FCM and DS & $\begin{array}{c}\text { Homogeneity and DS } \\
\text { (proposed method) }\end{array}$ \\
\hline Image 1 & & Sensitivity segmentation (\%) & 94.23 \\
Image 2 & 86.74 & 89.45 & 97.27 \\
Image 3 & 61.82 & 88.92 & 90.84 \\
Image 4 & 73.76 & 87.25 & 98.11 \\
Image 5 & 89.21 & 96.68 & 96.85 \\
Image 6 & 78.62 & 90.15 & 98.58 \\
Image 7 & 72.33 & 87.78 & 98.36 \\
Image 8 & 73.64 & 96.88 & 98.37 \\
Image 9 & 61.48 & 88.79 & 99.85 \\
Image 10 & 73.38 & 99.63 & 96.97 \\
Image 11 & 64.42 & 79.58 & 81.13 \\
Image 12 & 44.93 & 69.07 & 97.72 \\
\hline
\end{tabular}

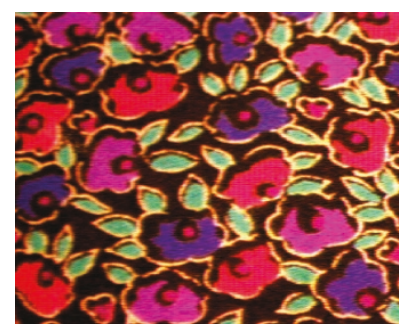

(a)

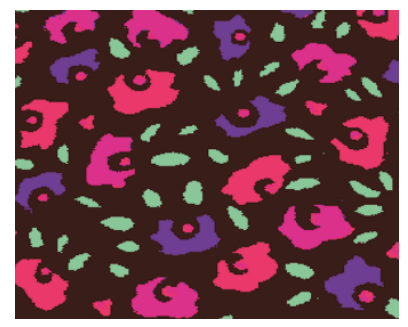

(c)

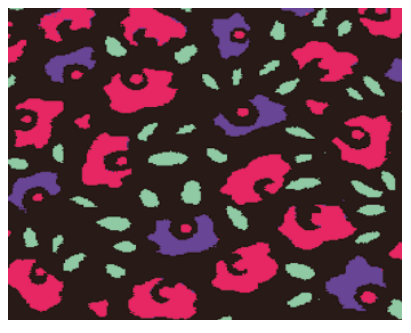

(b)

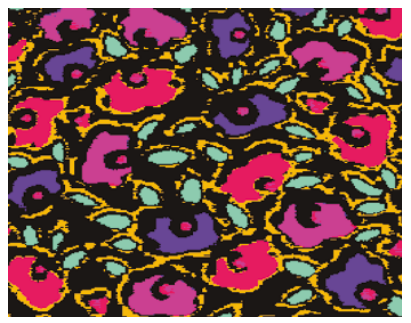

(d)
FIGURE 4: Comparison of the proposed segmentation method with other existing methods on a synthetic image (6 classes). (a) Original image $(256 \times 256 \times 3)$ : colour synthetic image with RGB description, (b) segmentation based on HCM and DS, (c) segmentation based on FCM and DS, and (d) segmentation based on homogeneity and DS.

Comparing Figures 4(b), 4(c), and 4(d), one can see that the different objects of the image are much better segmented in (d) than those in (b) and (c).

Figures 6 and 7 show other comparison results on a complex medical image. The segmentation results are obtained using the HCM, the FCM and Homogeneity method.

They correspond, respectively, to Figures 6(b), 6(c), and 6(d) in Figure 6. The cells are exactly and homogeneously segmented in Figure 6(d), which is not the case of Figures 6(b) and 6(c).

To evaluate the performance of the proposed segmentation algorithm, its accuracy was recorded.
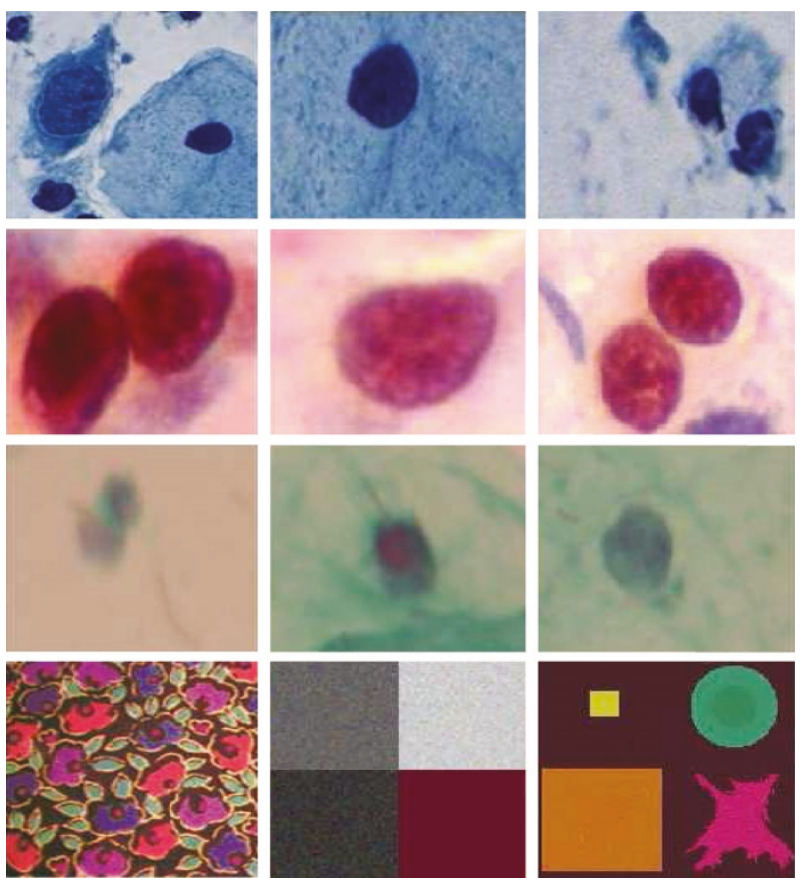

Figure 5: Data set used in the experiment. Twelve were selected for a comparison study. The patterns are numbered from 1 through 12, starting at the upper left-hand corner.

Regarding the accuracy, Tables 1 and 2 list the segmentation sensitivity of the different methods for the data set used in the experiment.

The segmentation sensitivity $[33,34]$ is determined as follows:

$$
\text { Sens }=\frac{N_{\mathrm{pcc}}}{N \times M} \times 100,
$$

with Sens, $N_{\mathrm{pcc}}, N \times M$ correspond, respectively, to the segmentation sensitivity (\%), number of correctly classified pixels and dimension of the image. The acquisition of the correct classified pixels is not a manual process; hence 


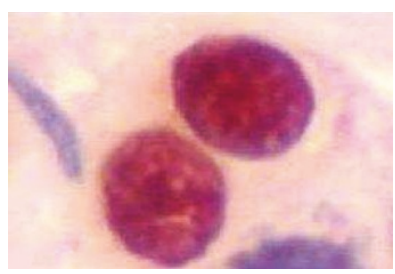

(a)

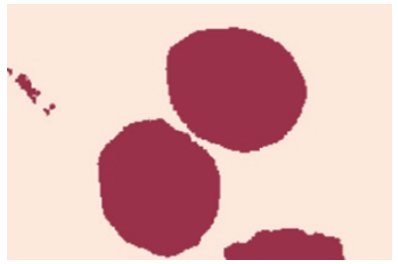

(c)

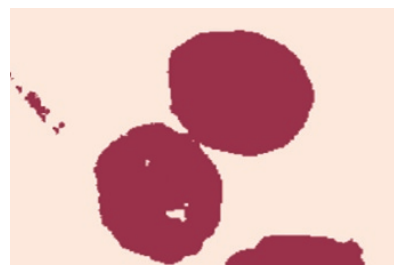

(b)

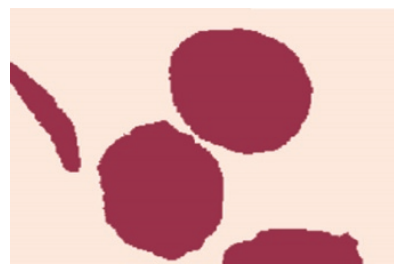

(d)
FIGURE 6: Comparison of the proposed segmentation method with other existing methods on a complex medical image (2 classes, various cells). (a) Original image $(256 \times 256 \times 3)$ : colour medical image with RGB description, (b) segmentation based on HCM and DS, (c) segmentation based on FCM and DS, and (d) segmentation based on homogeneity and DS.

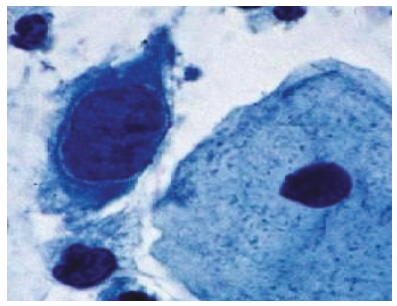

(a)

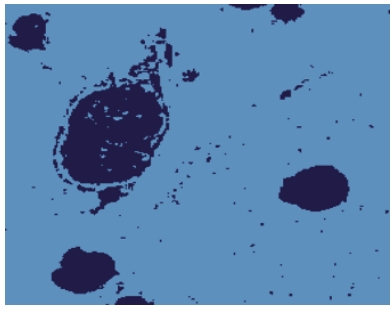

(c)

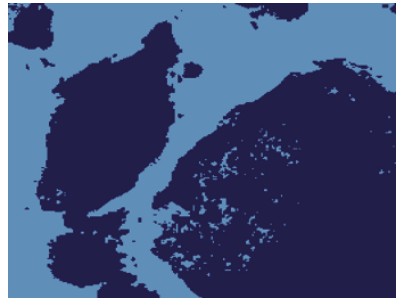

(b)

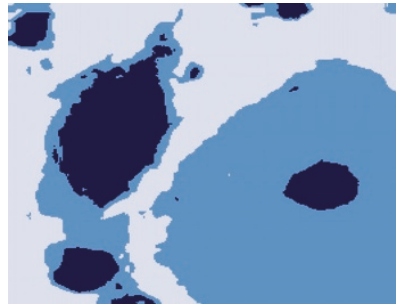

(d)
FIGURE 7: Comparison of the proposed segmentation method with other existing methods on a complex medical image (3 classes, various cells). (a) Original image $(256 \times 256 \times 3)$ : colour cells image with RGB description, (b) segmentation based on HCM and DS, (c) segmentation based on FCM and DS, and (d) segmentation based on homogeneity and DS.

software based on a reference image is run. It consists of a small program which compares the labels of the obtained pixels and the reference pixels as shown in Figure 3(f). The correctly classified pixel denotes a pixel with a label equal to its corresponding pixel in the reference image. The labeling of the original image is generated by the user based on the image used for segmentation.

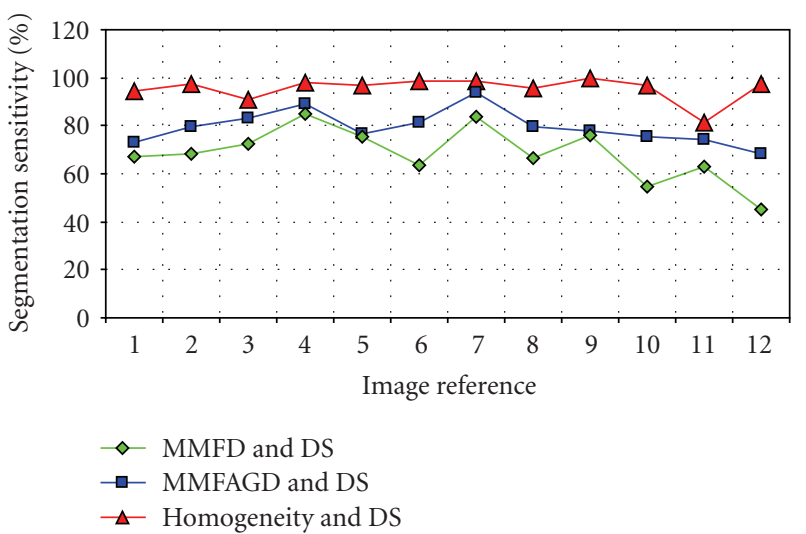

FIGURE 8: Segmentation sensitivity plots using MMFD and DS, MMFAGD and DS and homogeneity method and DS for the data set shown in Figure 5.

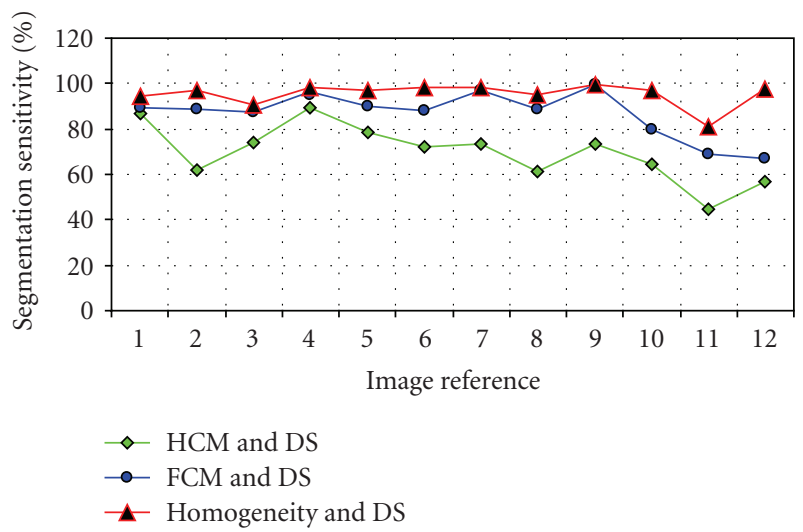

FIGURE 9: Segmentation sensitivity plots using HCM and DS, FCM and DS and homogeneity method and DS for the data set shown in Figure 5 .

In fact, the experimental results presented in Figure 6(d) are quite consistent with the visualized colour distributions in the objects, which make it possible to do an accurate measurement of cell volumes.

Parts (b), (c), and (d) of Figure 7 show other segmentation results and were obtained using HCM, FCM algorithms, and the homogeneity method, as used for the DS mass determination.

In Figure 7(a), only three colours are needed to represent the colour image (dark blue, blue, and background). In Figures $7(\mathrm{~b})$ and $7(\mathrm{c})$, the resulting image has only two colours. In Figure 7(d), the resulting image has three colours.

The partition resulting by the HCM is less accurate, and the partition resulting by FCM is not satisfactory either.

The performance of the homogeneity method is quite acceptable. In fact, one can observe in Figures 7(b) and 7 (c) that $13.26 \%$ and $10.55 \%$ of pixels were incorrectly segmented for the HCM and FCM methods, respectively. However, this demonstrates that the mass functions resulting from the two algorithms, do not always correspond to the intuitive concept of degree of belonging or compatibility, 
and the generated mass functions do not have a typical interpretation. Moreover, the HCM and FCM algorithms are instable in noisy environments. However, errors were largely reduced when exploiting simultaneously the three images through the use of the DS fusion method including the homogeneity histogram.

Indeed, only $5.77 \%$ of pixels were incorrectly segmented in Figure 7(d). This good performance between these methods can also be easily assessed by visually comparing the segmentation results.

The segmentation sensitivity values reported in Tables 1 and 2 are plotted in Figures 8 and 9, respectively.

Figure 8 shows two segmentation sensitivity plots using traditional methods such as MMFD and MMFAGD compared with the proposed method plot.

Figure 9 shows two other segmentation sensitivity plots using automatic methods such as HCM and FCM compared with the proposed method plot.

As seen on both Figures 8 and 9, the proposed method plot is clearly located on the top of the other methods plots.

Referring to segmentation sensitivity plots given in Figure 9, one observes that $27.67 \%, 12.22 \%$, and $1.42 \%$ of pixels were incorrectly segmented in Figures 6(b), 6(c), and 6(d), respectively. Comparing Figures 6(b) and 6(c) with Figure 6(d), the resulting image by the proposed method is much clearer than the one given by the HCM and FCM methods.

\section{Conclusion}

In this paper, we have proposed a new method for colour image segmentation based on homogeneity histogram thresholding and data fusion techniques. In the first phase, uniform regions are identified in each primitive colour via a thresholding operation on a newly defined homogeneity histogram. Then, the DS combination rule and decision are applied to fuse the three primitive colours.

The results obtained show the generic and robust character of the method in the sense that the local and global information were involved in the fusion process. On the other hand, in the estimation of mass function, we have used the local and global information. The results obtained demonstrated the significant improved performance in segmentation. The proposed method can be useful for colour image segmentation.

Nevertheless, there are some drawbacks to our proposed method. The used image models are mainly based on some a priori knowledge such as the mean and the standard deviation of each region of the image to be segmented. Also, in all our work, we have considered only one image for each application, whereas, many realizations of the same image fused together may be very helpful to the segmentation process. Furthermore, the research of other optimal models to estimate the mass functions in the Dempster-Shafer evidence theory and the fusion of imperfect information coming from different colour images are an important aspect of our present work. Also, the proposed method assumes that we have a reference image, which should be labelled by the user for comparison purposes. In practice, this is not realisable; hence advanced intelligent software for classification based on the Kohonen Neural Network may be used in parallel with the proposed segmentation procedure to avoid the manually labelling of the image by the user.

\section{References}

[1] E. Navon, O. Miller, and A. Averbuch, "Color image segmentation based on adaptive local thresholds," Image and Vision Computing, vol. 23, no. 1, pp. 69-85, 2005.

[2] S. Kasaei and M. Hasanzadeh, "Fuzzy image segmentation using membership connectedness," EURASIP Journal on Advances in Signal Processing, vol. 2008, Article ID 417293, 13 pages, 2008.

[3] H. D. Cheng, X. H. Jiang, Y. Sun, and J. Wang, "Color image segmentation: advances and prospects," Pattern Recognition, vol. 34, no. 12, pp. 2259-2281, 2001.

[4] R. Etienne-Cummings, P. Pouliquen, and M. A. Lewis, "A vision chip for color segmentation and pattern matching," EURASIP Journal on Applied Signal Processing, vol. 2003, no. 7, pp. 703-712, 2003.

[5] X. Gao, K. Hong, P. Passmore, L. Podladchikova, and D. Shaposhnikov, "Colour vision model-based approach for segmentation of traffic signs," EURASIP Journal on Image and Video Processing, vol. 2008, Article ID 386705, 7 pages, 2008.

[6] R. Bradley, "A unified Bayesian decision theory," Theory and Decision, vol. 63, no. 3, pp. 233-263, 2007.

[7] I. Bloch and H. Maitre, "Fusion of image information under imprecision," in Aggregation and Fusion of Imperfect Information, B. Bouchon-Meunier, Ed., Series studies in fuzziness, Physical Verlag, pp. 189-213, Springer, 1997.

[8] S.-L. Dong, J.-M. Wei, T. Xing, and H.-T. Liu, "Constraintbased fuzzy optimization data fusion for sensor network localization," in Proceedings of the 2 nd International Conference on Semantics Knowledge and Grid (SKG '06), p. 59, November 2006.

[9] C. Lucas and B. N. Araabi, "Generalization of the DempsterShafer theory: a fuzzy-valued measure," IEEE Transactions on Fuzzy Systems, vol. 7, no. 3, pp. 255-270, 1999.

[10] D. Dubois and H. Prade, "Possibility theory and its applications: a retrospective and prospective view," in Proceedings of the IEEE International Conference on Fuzzy Systems, vol. 1, pp. 5-11, May 2003.

[11] A. P. Dempster, "Upper and lower probabilities induced by multivalued mapping," Annals of Mathematical Statistics, vol. 38, pp. 325-339, 1967.

[12] G. Shafer, A Mathematical Theory of Evidence, Princeton University Press, 1976.

[13] T. Denœux, "A k-nearest neighbor classification rule based on Dempster-Shafer theory," IEEE Transactions on Systems, Man \& Cybernetics, vol. 25, no. 5, pp. 804-813, 1995.

[14] H.-J. Zimmermann and P. Zysno, "Quantifying vagueness in decision models," European Journal of Operational Research, vol. 22, no. 2, pp. 148-158, 1985.

[15] K. Raghu and J. M. Keller, "A possibilistic method to clustering," IEEE Transactions on Fuzzy Systems, vol. 1, no. 2, 1993.

[16] P. Vannoorenberghe, O. Colot, and D. De Brucq, "Color image segmentation using Dempster-Shafer's theory," IEEE International Conference on Image Processing (ICIP '99), vol. 4, pp. 300-304, October 1999. 
[17] Y. M. Zhu, L. Bentabet, O. Dupuis, V. Kaftandjian, D. Babot, and M. Rombaut, "Automatic determination of mass functions in Dempster-Shafer theory using fuzzy c-means and spatial neighborhood information for image segmentation," Optical Engineering, vol. 41, no. 4, pp. 760-770, 2002.

[18] R. R. Yager, "Class of fuzzy measures generated from a Dempster-Shafer belief structure," International Journal of Intelligent Systems, vol. 14, no. 12, pp. 1239-1247, 1999.

[19] J. C. Bezdek, Pattern Recognition with Fuzzy Objective Function Algorithms, Plenum Press, New York, NY, USA, 1981.

[20] M. N. Ahmed, S. M. Yamany, N. Mohamed, A. A. Farag, and T. Moriarty, "A modified fuzzy C-means algorithm for bias field estimation and segmentation of MRI data," IEEE Transactions on Medical Imaging, vol. 21, no. 3, pp. 193-199, 2002.

[21] R. Duda and P. Hart, Pattern Classification and Scene Analysis, John Wiley \& Sons, New York, NY, USA, 1973.

[22] L. Gautier, A. Taleb-Ahmed, M. Rombaut, J. G. Postaire, and H. Leclet, "Help to decison of segmentation of pictures by Dempster-Shafer theory: application to MRI sequence," Editions Scientifiques et Medicales Elsevier SAS, vol. 22, no. 6, pp. 378-392, 2001.

[23] S. Ben Chaabane, M. Sayadi, F. Fnaiech, and E. Brassart, "Dempster-Shafer evidence theory for image segmentation: application in cells images," International Journal of Signal Processing, vol. 5, no. 1, 2009.

[24] S. Ben Chaabane, M. Sayadi, F. Fnaiech, and E. Brassart, "Color image segmentation based on Dempster-Shafer evidence theory," in Proceedings of the Mediterranean Electrotechnical Conference (MELECON '08), pp. 862-866, 2008.

[25] H. D. Cheng, C. H. Chen, H. H. Chiu, and H. Xu, "Fuzzy homogeneity approach to multilevel thresholding," IEEE Transactions on Image Processing, vol. 7, no. 7, pp. 1084-1088, 1998.

[26] E. Littmann and H. Ritter, "Adaptive color segmentation-a comparison of neural and statistical methods," IEEE Transactions on Neural Networks, vol. 8, no. 1, pp. 175-185, 1997.

[27] M. Sezgin and B. Sankur, "Survey over image thresholding techniques and quantitative performance evaluation," Journal of Electronic Imaging, vol. 13, no. 1, pp. 146-168, 2004.

[28] H.-D. Cheng and Y. Sun, "A hierarchical approach to color image segmentation using homogeneity," IEEE Transactions on Image Processing, vol. 9, no. 12, pp. 2071-2082, 2000.

[29] H. D. Cheng, X. H. Jiang, and J. Wang, "Color image segmentation based on homogram thresholding and region merging," Pattern Recognition, vol. 35, no. 2, pp. 373-393, 2002.

[30] R. C. Gonzalez and P. Wintz, Digital Image Processing, Addison-Wesley, Reading, Mass, USA, 1987.

[31] M. Heath, S. Sarkar, T. Sanocki, and K. Bowyer, "Comparison of edge detectors: a methodology and initial study," in Proceedings of the IEEE Computer Society Conference on Computer Vision and Pattern Recognition, pp. 143-148, 1996.

[32] G. Liu and R. M. Haralick, "Assignment problem in edge detection performance evaluation," in Proceedings of the IEEE Computer Society Conference on Computer Vision and Pattern Recognition, vol. 1, pp. 26-31, 2000.

[33] V. Grau, A. U. J. Mewes, M. Alcañiz, R. Kikinis, and S. K. Warfield, "Improved watershed transform for medical image segmentation using prior information," IEEE Transactions on Medical Imaging, vol. 23, no. 4, pp. 447-458, 2004.

[34] R. O. Duda, P. E. Hart, and D. G. Sork, Pattern Classification, Wiley-Interscience, New York, NY, USA, 2000. 